\title{
Evaluating the Effects of Model Generalization on Intrusion Detection Performance
}

\author{
Zhuowei $\mathrm{Li}^{12}$, Amitabha Das ${ }^{2}$ and Jianying Zhou ${ }^{3}$ \\ 1 Indiana University, USA. zholi@indiana edu \\ 2 Nanyang Technological University, Singapore. asadas@ntu.edu.sg \\ 3 Institute of Infocomm Research, Singapore. jyzhou@i2r.a-star.edu.sg
}

\begin{abstract}
An intrusion detection system usually infers the status of an unknown behavior from limited available ones via model generalization, but the generalization is not perfect. Most existing techniques use it blindly (or only based on specific datasets at least) without considering the difference among various application scenarios. For example, signature-based ones use signatures generated from specific occurrence environments, anomaly-based ones are usually evaluated by a specific dataset. To make matters worse, various techniques have been introduced recently to exploit too stingy or too generous generalization that causes intrusion detection invalid, for example, mimicry attacks, automatic signature variation generation etc. Therefore, a critical task in intrusion detection is to evaluate the effects of model generalization. In this paper, we try to meet the task. First, we divide model generalization into several levels, which are evaluated one by one to identify their significance on intrusion detection. Among our experimental results, the significance of different levels is much different. Under-generalization will sacrifice the detection performance, but over-generalization will not lead to any benefit. Moreover, model generalization is necessary to identify more behaviors in detection, but its implications for normal behaviors are different from those for intrusive ones.
\end{abstract}

\section{Introduction}

There exist two general approaches for detecting intrusions: signature-based intrusion detection (SID, a.k.a. misuse detection), where an intrusion is detected if its bchavior matches existing intrusion signatures, and anomaly-based intrusion detection (AID), where an intrusion is detected if the resource behavior deviates from normal behaviors significantly. From another aspect, there are two behavior spaces for intrusion detection (Figure 1): normal behavior space and intrusive behavior space, and they are complementary to each other. Conceptually, SID is based on knowledge in intrusive behavior space, and AID is based on knowlcdge in normal behavior space [2]. Perfect detection of intrusions can be achicved only if we have a completc model of any onc of the two behavior spaces, because what is not bad is good and vice versa ideally. Figure 1 (a) and (b) illustrate the behavior models for SID (i.e., intrusive behavior model) and for AID (i.e., normal behavior model) in the real applications.

A critical problem. There are two quality factors within the behavior models: inaccuracy and incompleteness. For example, a part of the intrusive behavior

Please lise the following format when citing this chapter:

Li, Z., Das, A., and Zhou, J., 2007, in IFIP International Federation for Information Processing, Volume 232.. New Approaches for Security, Privacy and Trust in Complex Environments, eds. Venter, H., Eloff, M., Labuschagne, L., Eloff, J.n von Solms, R., (Boston: Springer), pp. 421-432. 


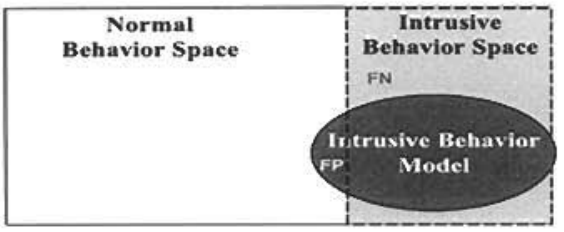

(a) Intrusive Behavior Model.

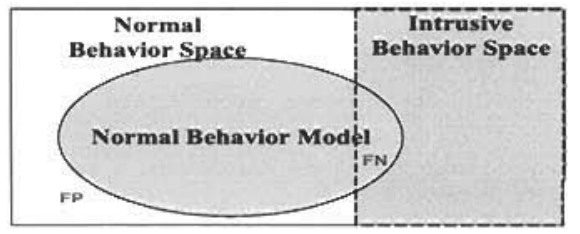

(b) Normal Behavior Model.

Fig. 1. Behavior spaces and models.

model falling into the normal behavior space leads to the inaccuracy. Due to incompleteness, the intrusive behavior model cannot cover all intrusive behavior space, and the normal behavior model cannot cover all the normal behavior space either. In SID (Figure 1.a), model inaccuracy will lead to false positives (FP) and model incompleteness in it will lead to false negatives (FN). In contrast, model inaccuracy in the normal bchavior model will lead to FNs and model incompleteness in it will cause FPs (Figure 1.b). To build a practical intrusion detection system, it is critical to reduce the model inaccuracy and incompleteness, and thus to lower FPs and FNs in the detection phase.

Past addressings. To make up for the incompleteness, most existing 'model building' techniques try to infor the unknown behaviors via model generalization (defined in Section 3), which is able to climinate FNs in SID and to reduce FPs in AID. However, as indicated in Figure 1, it can also lead to more FPs in SID and more FNs in AID. In other words, model generalization is twoedged for intrusion detection in principle [9]. Various techniques have been introduced recently to exploit too stingy or too gencrous model generalization (Section 2), for example, mimicry attacks[11], mutate exploits[10], automatic signature variation gencration[7] ctc.

Evaluation. Thus, it is very useful to identify the utility of model generalization. We can envision at least four of its applications.

Determine deployment conditions for an intrusion detection technique, as well as proper techniques to detect intrusions into a specific environment.

Guide the development of an adaptive intrusion detection technique by adjusting the gencralization cxtent.

Alleviate concept drifting. Intrusion and application cvolution patterns can determine the extent of generalization in an ad hoc deployment.

- Perform intrusion detection evaluation. According to different generalization extents, we can generatc appropriate artificial datasets, which can identify the generic detection capability of a SID/AID technique.

Our contributions. We believe that our evaluation advances the rescarch on intrusion detection in two perspectives. First, we design a framework to evaluate the effect of model generalization, in which model generalization is achicved at different levels according to the reasonableness of the underlying assumptions. Sccondly, on a typical dataset, our experiments are performed to verify the cvaluation framework, and to identify the utility of model gencralization. 
The remaining parts are organized as follows. Section 2 reviews the related work on model generalization. In section 3, an evaluation framework for model generalization is designed. As a case study, experiments in section 4 reveal the implications of model generalization on intrusion detection. Lastly, we draw conclusions and lay out the future work in section 5 .

\section{Related Work}

To our knowledge, we are the first to evaluate model generalization for intrusion detection while there are two existing implicit applications of model generalization: extending behavior models and evade detection.

First, the intrusion signatures can be generalized to cover more intrusion variations. Anchor et al. [1] applied the evolutionary programming to optimize the generalization in an intrusion signature, and thus to detect more intrusion variants. Rubin et al. [8] presented a method to construct more robust signatures from existing intrusion signatures. Secondly, the normal behavior model of AID can be generalized as well. In [5, 12], existing audit trails are modeled inexactly to accommodate more behaviors, and thus to achicve model gencralization.

Several work is proposed to utilize the false negatives introduced by model generalization. In AID techniques, mimicry attacks [11] are designed to misuse the generalization by mimicking its normal behaviors, and thus to avoid being detected. In SID techniques, model generalization is also exploited $[10,7]$ to generate intrusion variations, which cannot be detected either.

In summary, too generous generalization in AID will make mimicry attacks successful [11], while too stingy gencralization in SID will make some attack variations undetectable $[8,10]$. In our research, we try to identify the relations between the extent of generalization and detection performance.

\section{An Evaluation Framework for Model Generalization}

In this section, we proposed the evaluation framework for model generalization bascd on a theoretical basis for intrusion detection $[6]$.

\subsection{Theoretical Basis for Intrusion Detection}

In a nutshell, the basis introduces three new concepts to formalize the process of intrusion detection: feature range, NSA label and compound feature. Every instance in a training audit trail can be represented as a feature range of a highorder compound feature, and every feature range has a NSA label, which is used to detect behaviors in test audit trails. In detail, the value of every feature in an instance can be replaced with a feature range, which is gotten by extending its value so that the extension does not conflict with other existing values. The feature ranges of all features are compounded using cartesian products to build a (training or test) behavior signature for intrusion detection.

In this framework, it is supposed that there is a training audit trail and a feature vector $F V=\left\{F_{1}, F_{2}, \ldots, F_{n}\right\}$. For every feature $F_{i}$, a series of feature ranges $R_{F_{2}}^{1}, R_{F_{2}}^{2}, \ldots, R_{F_{2}}^{m}$ is first mined from the training audit trails. Using feature ranges of all features, the behavior signatures $S i g_{1}, S i g_{2} \ldots, S_{i g_{l}}$ are 
constructed for intrusion detection. In the detection phase, a test instance is formalized as a signature $\mathrm{Sig}_{t}$, and it is detected in accordance with whether it matches any existing behavior signature.

\subsection{Model Generalization}

We first define model generalization within the context of intrusion detection.

Definition 1 (Model Generalization). Suppose that there exists a set of behaviors associated with a resource. Model generalization is an operation that tries to identify a new behavior associated with the same resource based on the existing set of behavior instances.

Model generalization can improve the detection ratc by identifying more novel behaviors (e.g., normal behaviors) but may also degrade the detection performance by mis-identifying novel behaviors because of generalization errors [9]. This influence of model generalization on detection performance is generally determined by its underlying assumptions per se. In our evaluation, we first pinpoint three phases of our framework where we can use various assumptions to apply three levels of generalization, and then evaluate them one by one for model generalization. We also include a level without any generalization in which the bchaviors in the training audit trails are represented precisely.

In the follow-up subsections, we describe the methods to evaluate the three levels of generalizations which moves the model from most specialized to most generalized as we move down the level (from L0 to L3).

\subsection{Lo Without Generalization}

Suppose that for a feature $F$, there cxists a series of feature values, $v_{1}, v_{2}, \ldots, v_{l}$. Without generalization, every feature value $v_{i}$ is regarded as a feature range with its upper and lower bounds equal to $v_{i}$. In this way, the instances in the training audit trails are represented precisely by the signatures generated from these feature ranges. Note that, for $F$, we have not inferred the NSA label of unknown feature subspace between any two feature values.

\subsection{L1 Model Generalization}

For every feature, to achieve L1 generalization, we assume that the unknown parts in its feature space have the same NSA label as its neighboring feature values. Obviously, inherent in this assumption is a concept of distancc. Therefore, due to the lack of distance concept in nominal features, we will only discuss the L1 generalization on numerical (discrete and continuous) features, and regard every feature value of a nominal feature as a feature rangc. For convenience, we use two more notations on a feature range $R_{F}^{i}: U p p\left(R_{F}^{i}\right)$ is its upper bound and $\operatorname{Low}\left(R_{F}^{2}\right)$ is its lower bound. With respect to a feature value $v_{i}$, an initial feature range $R_{F}^{i}$ will be formed with $\operatorname{Upp}\left(R_{F}^{i}\right)=\operatorname{Low}\left(R_{F}^{i}\right)=v_{i}$.

L1 generalization is described in algorithm 1 . In this generalization, one critical step is to split the unknown subspace $\left(v_{i}, v_{i+1}\right)=\left(U p p\left(R_{F}^{i}\right), \operatorname{Low}\left(R_{F}^{i+1}\right)\right)$ $(i+1 \leq l)$, and allocate the two parts to existing neighboring ranges $R_{F}^{i}$ and $R_{F}^{i+1}$. We use several strategies and evaluate them in our framework. These are: 


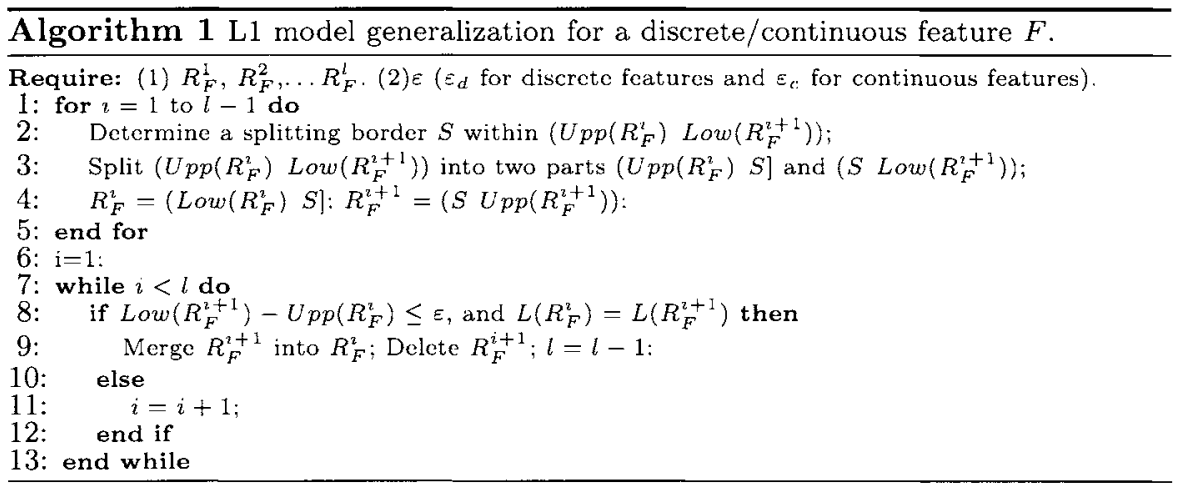

(1) no splitting (2) equal splitting, (3) frequency-based splitting, (4) intrusionspecific splitting. Note that, in Algorithm 1, the merging step for feature ranges (i.e., lines 6-12) is selective after the splitting step (i.e., lines 1-5). This step for merging range is also a generalization operation in L1 generalization.

1. L1.1: No splitting. If we do not conduct the merging step either, the L1.1 generalization actually becomes same as LO, i.e., no generalization.

2. L1.2: Splitting it equally. The unknown interval between $v_{i}$ and $v_{i+1}$ is split at the midpoint $S=\frac{v_{2}+v_{i+1}}{2}$. That is, $\left(v_{i}, S\right]$ is assigned the same NSA label as $v_{i}$, and $\left(S, v_{i+1}\right)$ is assigned the same NSA label as $v_{i+1}$.

3. L1.3: Frequency-based Splitting. Let the frequency of $v_{i}$ in the training audit trails be $f_{v_{i}}$. Then, the splitting point is $S=v_{i}+\left(v_{i+1}-v_{i}\right) * \frac{f_{v_{i}}}{f_{v_{2}}+f_{v_{2}+1}}$. $\left(v_{i}, S\right]$ is assigned as $L\left(v_{i}\right)$, and $\left(S, v_{i+1}\right)$ is assigned as $L\left(v_{i+1}\right)$.

4. L1.4: Intrusion specific splitting. Given a predefined generalization parameter $G_{i n}$ for intrusions. For a pair of neighboring values $v_{i}$ and $v_{i+1}$, if $L\left(v_{i}\right)=\mathrm{N}$ and $L\left(v_{i+1}\right)=\mathrm{A}, S=v_{i+1}-G_{i n}$. If $L\left(v_{i}\right)=\mathrm{A}$ and $L\left(v_{i+1}\right)=\mathrm{N}$, $S=v_{i}+G_{i n}$. Otherwise, $S=\frac{v_{2}+v_{i+1}}{2} .\left(v_{i}, S\right]$ is assigned as $L\left(v_{i}\right)$, and $\left(S, v_{\imath+1}\right)$ is assigned as $L\left(v_{i+1}\right)$.

In addition, we also evaluate the merging step for every splitting strategy.

In the detection phase, every instance is formalized as $S i g_{t}$ by replacing every value with its feature range. Finally, we evaluate whether Sig $_{t}$ matches any signature in $\Omega\left(\begin{array}{ll}F_{1} & n\end{array}\right)$. If matched, it is identified by that signature. Otherwise, $\mathrm{Sig}_{t}$ will further be evaluated by $\mathrm{L} 2$ generalization evaluation processes.

\subsection{L2 Model Generalization}

After the L1 model generalization, all the (nominal, discrete, and/or continuous) features are uniformly represented by a series of feature ranges. In L 2 model generalization, we will utilize the relations between feature ranges rather than values, which are measured by the distance of two signatures. To this end, let us first define a distance function of two signatures in the bchavior models.

Signature distance. Let $R\left(S_{i g}, F_{i}\right)$ denote the feature range of $F_{i}$ in a signature $S i g_{1}$. For any two signatures, $S i g_{1}$ and $S i g_{2}$, their distance is: 


$$
D\left(S i g_{1}, S i g_{2}\right)=\sum_{i=1}^{n} \delta\left(S i g_{1}, S i g_{2}, F_{i}\right)
$$

Where, $\delta\left(\operatorname{Sig}_{1}, \operatorname{Sig}_{2}, F_{i}\right)=\left\{\begin{array}{l}0, \text { if } R\left(\operatorname{Sig}_{1}, F_{i}\right)=R\left(\operatorname{Sig}_{2}, F_{i}\right) \\ 1, \text { otherwise. }\end{array}\right.$

Evaluating L2 generalization. L2 gencralization is achieved by the following two generalization opcrations. L2.1: grouping feature ranges. If several feature ranges of a feature are interchangeable in $\Omega\left(F_{1} n\right)$ without loss of signature distinguishability, they will be combined into a group. L2.2: mutating feature ranges. For a feature, its feature range in a signature can be mutated to any of its other feature ranges without loss of signature distinguishability.

Grouping feature ranges. For a feature $F_{i}$, if a feature range in $\Omega\left(\begin{array}{ll}F_{1} & n\end{array}\right)$ is interchangeable with another feature range without loss of signature distinguishability (i.e. without changing its NSA label), their significance is equal to each other. We can group these feature ranges in constructing behavior models. As a special case, a feature range can form a group by itself. In this way, we can form a scries of groups for $F_{i}, G_{F_{i}}=\left\{G_{F_{i}}^{1}, G_{F_{i}}^{2}, \ldots\right\}$ such that for any feature range $R_{F_{z}}^{j}$, there is a group $G_{F_{2}}^{k}, R_{F_{z}}^{j} \in G_{F_{z}}^{k}$. Finally, we achieve a grouping scheme for all features in the feature vector: $G_{F V}=\left\langle G_{F_{1}}, G_{F_{2}}, \ldots, G_{F_{n}}\right\rangle$.

For two signatures $S i g_{1}$ and $S i g_{2}$ in $\Omega\left(F_{1} n\right)$, they are equivalent to each other with respect to $G_{F V}$ based on the following rule.

$$
\begin{aligned}
& S_{i g_{1}} \stackrel{G_{F} V}{=} S_{i g_{2}} \Leftrightarrow \exists i\left(\delta\left(S_{i g_{1}}, S i g_{2}, F_{i}\right)=1\right) \\
& \wedge\left(\exists j\left\{R\left(S i g_{1}, F_{i}\right), R\left(S i g_{2}, F_{i}\right)\right\} \subset G_{F_{i}}^{j}\right)
\end{aligned}
$$

For any two equivalent signatures, they are compatible if they have the same NSA label. Otherwise, they are conflict to each other in the behavior models.

The behavior models can be generalized by grouping feature ranges. For cxample, for signatures " $\langle a, 1, E\rangle$ " and " $\langle b, 2, F\rangle$ ", if ' $a$ ' and 'b' are grouped, the behavior models can be enlarged by two additional signatures " $\langle b, 1, E\rangle$ " and " $\langle a, 2, F\rangle "$. Essentially, like in Genetic Algorithm [4] we arc allowing crossover operation between signatures by interchanging the feature ranges in a group.

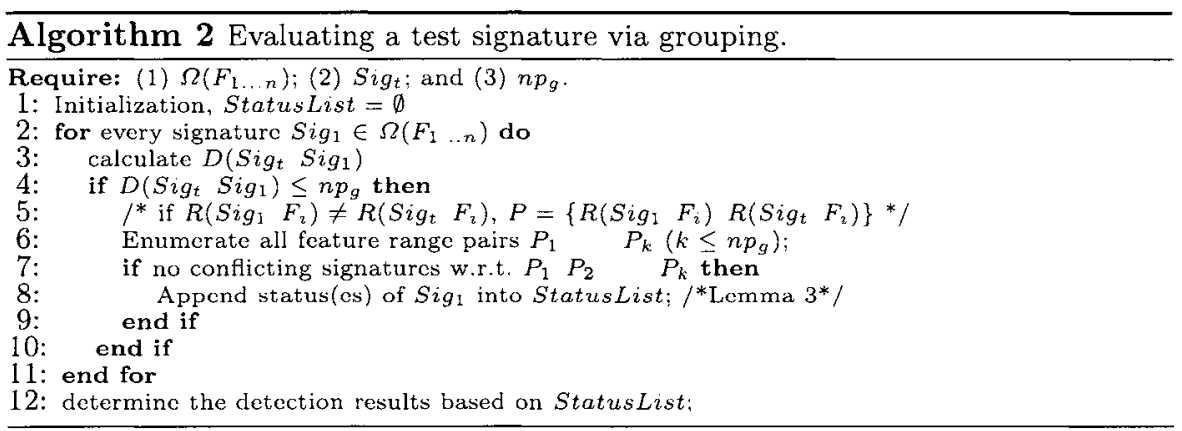

Moreover, to measure the diversity in $G_{F V}$, the number of grouping points $n p_{g}$ is utilized in the detection phase. In other words, if the grouping scheme 
does not exist, there are at least $n-n p_{g}$ equivalent feature ranges between $\mathrm{Sig}_{t}$ and any signature $\mathrm{Sig}_{i}$ in the behavior models. The larger the parameter $n p_{g}$ is, the more diverse the group operation is. Given $n p_{g}$ and $\Omega\left(F_{1} n\right)$, a test instance is evaluated as in Algorithm 2.

If the output is an anomaly, we will evaluate $S_{i g}$ using mutation operation.

Mutating feature ranges. Neglecting some features will cause a signature to identify more behaviors. For example, suppose that there is a signature "height $\in(156 \mathrm{~cm}, 189 \mathrm{~cm}]$, weight $\in(45 \mathrm{~kg}, 75 \mathrm{~kg})$, and Nationality $=U S A$ ". If all threc features are used, it cannot identify the instance 'height $=174 \mathrm{~cm}$, weight $=$ $65 \mathrm{~kg}$, and Nationality = China' will not be identified. But if 'Nationality' is ignored, the signature will identify the instance. Essentially, ignoring features is equal to the mutation operation in Genetic Algorithms[4]. One condition of the mutation is that it should not lead to any contradiction in the existing signatures. For example, if we let $F_{1}$ and $F_{2}$ mutate, signatures " $\langle a, b, c, d\rangle$ " in $N\left(\begin{array}{ll}F_{1} & 4\end{array}\right)$ and " $\langle x, y, c, d\rangle$ " in $A\left(\begin{array}{ll}F_{1} & 4\end{array}\right)$ will contradict to each other.

Furthermore, we use a mutation point number $n p_{m}$ to measure the diversity of the mutation process. In the detection phase, given $n p_{m}$ and $\Omega\left(F_{1} n\right)$, the unidentified test signature $\mathrm{Sig}_{t}$ will be evaluated as in Algorithm 3 .

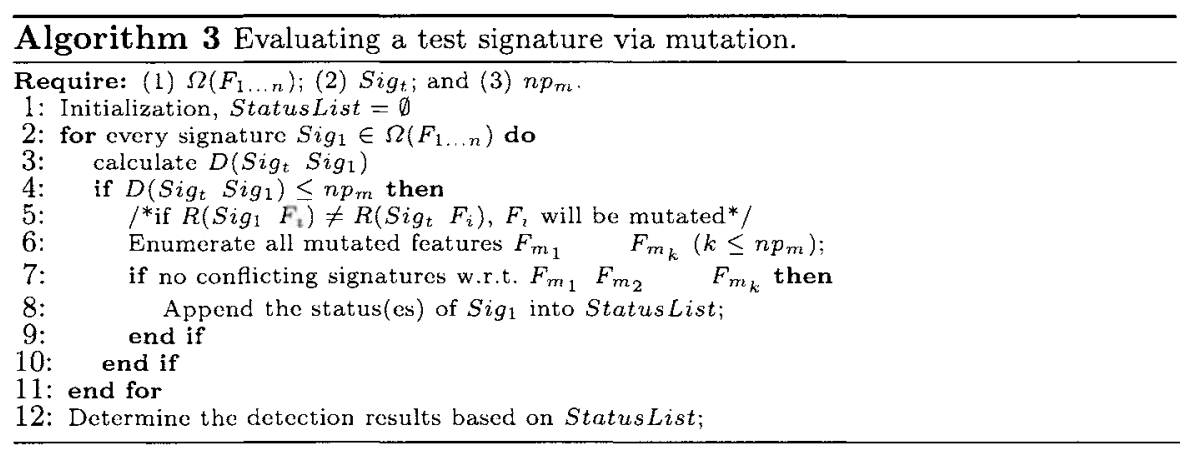

\subsection{L3 Model Generalization}

If the test signature $S i g_{t}$ cannot be identified by L1 and L2 generalization, it will be identified by the signature(s) with the minimum distance to it.

Nearest signatures. We assume that the test signature has the same NSA label as its nearest signature(s) in the behavior models, which is measured by its minimum distancc to all signatures in $\Omega_{F_{1 \ldots n}}$,

$$
D_{\min }\left(\operatorname{Sig}_{t}, \Omega\left(F_{1} \quad n\right)\right)=\min _{\operatorname{Sig}_{\imath} \in \Omega\left(F_{1 \ldots n}\right)} D\left(\operatorname{Sig}_{i}, S_{i g_{t}}\right)
$$

\subsection{Measuring the Detection Performance}

We assign a cost scheme as in Table 1 to quantify the detection performance, and calculate the average detection cost of an instance in the test audit trails. If the behavior is identified correctly, the cost is 0. Otherwise, we can assign some penalty for the detection result. In our cost scheme, we assume 
that the detection of an intrusion as an anomaly is useful but it is less useful than identifying an intrusion. Specifically, suppose that there are $T$ instances in the test audit trails. The number of false positives is $\#_{N A}$, and for false negatives, it is \#IN. The average cost of a test instance is defined

\begin{tabular}{|c|l|c|c|c|}
\hline INDEX & NOTATIONS & ORIGINAL Class & DETECTION RESULTS & COST \\
\hline 1 & $\#_{N N}$ & normal & normal & 0 \\
2 & $\#_{N A}$ & normal & anomaly & 3 \\
3 & $\#_{I I}$ & intrusion & original intrusion & 0 \\
4 & $\#_{I A}$ & intrusion & anomaly & 1 \\
5 & $\#_{I N}$ & intrusion & normal & 3 \\
\hline
\end{tabular}

Table 1. Detection results and their costs.

as: cost $=\#_{N A} \times 3+\#_{I N} \times 3+\#_{I A} \times 1 \times \frac{1}{T}$. In addition, the average cost in absence of any generalization gives the reference baseline, cost $t_{\text {base }}$, of the detection performance. In practice, the usefulness of model generalization is reflected in the relation between its average cost and cost $_{\text {base }}$. If cost $>$ cost $_{\text {base }}$, its performance has been degraded by such model generalization. Otherwise, the model generalization can be assumed to be useful for intrusion detection.

\section{Experiments: A Case Study}

We have chosen a typical dataset from KDD CUP 1999 contest [3], which mects the requirements of our framework: labeled audit trails and an intrusion-specific feature vector, in which $\varepsilon_{d}=1$ and $\varepsilon_{c}=0.01$. In order to keep the computation within reasonable limits, we sample instances from the datasets: 10000 instances from the total 4898431 training instances and 500 instances from 311029 test instances randomly. For convincing, we give three pairs of such training and test samples. We have performed our experiments on larger samples, but the experimental results on our larger samples have the same characteristics to the results on the current samples.

\subsection{Without Model Generalization}

Table 2 lists the detection results when there is no generalization, and they are

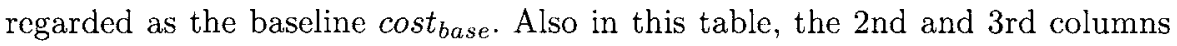
give the numbers of normal and intrusive instances in every sample pair.

\begin{tabular}{|l||c|c||c|c|c|c|c|c|}
\hline Sample & Norm. & Intru. & $\#_{N N}$ & $\#_{N A}$ & $\#_{I I}$ & $\#_{I A}$ & $\#_{I N}$ & cost \\
\hline Pair 1 & 103 & 397 & 0 & 103 & 203 & 193 & 1 & 1.01 \\
Pair 2 & 91 & 409 & 0 & 91 & 216 & 193 & 0 & 0.932 \\
Pair 3 & 108 & 392 & 5 & 103 & 193 & 198 & 1 & 1.02 \\
\hline
\end{tabular}

Table 2. L0: without model generalization.

Among the detection results, more than half of intrusive instances are identified correctly (denoted by $\#_{I I}$ ), but, in comparison, almost all normal instances are detected incorrectly. To some extent, it indicates that the normal behaviors are of great variety, and more generalization is needed to infer their statuses. 


\begin{tabular}{|c|c|c|c|c|c|c|c|c|c|c|c|c|c|}
\hline \multirow[b]{2}{*}{$\mathrm{L} 1$} & \multirow[b]{2}{*}{$G_{\imath n}$} & \multicolumn{6}{|c|}{ with the range merging step: } & \multicolumn{6}{|c|}{ without the range merging step: } \\
\hline & & $\# N N$ & $\# N A$ & $\#_{11}$ & \# I A & $\# I N$ & cost & $\#_{N N}$ & $\#_{N A}$ & $\#_{I I}$ & \#IA & $\#_{I N}$ & $\cos t$ \\
\hline $\mathrm{L} 1.4$ & 0 & 35 & 68 & 280 & 115 & 2 & 0.65 & 6 & 97 & 278 & 118 & 1 & $0 . \overline{824}$ \\
\hline $\mathrm{L} 1.4$ & 1 & 35 & 68 & 280 & 115 & 2 & 0.65 & 6 & 97 & 278 & 118 & 1 & 0.824 \\
\hline $\mathrm{L} 1.4$ & 2 & 35 & 68 & 281 & 114 & 2 & 0.648 & 6 & 97 & 278 & 118 & 1 & 0.824 \\
\hline L1.4 & 3 & 35 & 68 & 280 & 115 & 2 & 0.65 & 6 & 97 & 279 & 117 & 1 & 0.822 \\
\hline L1.4 & 4 & 35 & 68 & 281 & 114 & 2 & 0.648 & 6 & 97 & 279 & 117 & 1 & 0.822 \\
\hline L1.4 & 5 & 35 & 68 & 281 & 114 & 2 & 0.648 & 6 & 97 & 278 & 118 & 1 & 0.824 \\
\hline L1.4 & 10 & 35 & 68 & 280 & 115 & 2 & 0.65 & 6 & 97 & 279 & 117 & 1 & 0.822 \\
\hline L1.4 & 20 & 35 & 68 & 281 & 114 & 2 & 0.648 & 6 & 97 & 278 & 118 & 1 & 0.824 \\
\hline
\end{tabular}

Table 3. L1.4 generalization on the 1st sample pair.

\subsection{Evaluating L1 Model Generalization}

Table 3 gives the detection performance on the 1st sample pair with L1.4 generalization, where $G_{i n} \in\{0,1,2,3,4,5,10,20\}$. Obviously, the value of $G_{i n}$ has no influence on the detection performance in all aspects. The same phenomenon is held in the other two sample pairs of our experiments as well. Thus, we let $G_{i n}=0$ in the following experiments.

The utility of the range merging step. In Table 3, the range merging step has contributed much to the performance enhancement by identifying more normal behaviors. Note that the range merging step has little effect on the identification ability for intrusive behaviors.

Table 4 gives the evaluation results on the four scenarios of $L 1$ generalization. We analyze their utility for intrusion detection, and their difference.

\begin{tabular}{|c|c|c|c|c|c|c|c|c|c|c|c|c|}
\hline & \multicolumn{6}{|c|}{ with the range merging step } & \multicolumn{6}{|c|}{ without the range merging step } \\
\hline L1 & $\# N N$ & $\#_{N A}$ & $\# I I$ & $\# 1 \mathrm{~A}$ & \#IN & cost & $\#_{N N}$ & $\#_{N A}$ & $\#_{I I}$ & $\#$ IA & $\#$ IN & cost \\
\hline \multicolumn{13}{|c|}{ (Pair 1) Normal:Intrusion $=103: 397$} \\
\hline $\mathrm{L} 1.1$ & 6 & 97 & 241 & 155 & 1 & 0.898 & 0 & 103 & 203 & 193 & 1 & 1.01 \\
\hline $\mathrm{L} 1.2$ & 35 & 68 & 281 & 114 & 2 & 0.648 & 6 & 97 & 279 & 117 & 1 & 0.822 \\
\hline $\mathrm{L} 1.3$ & 35 & 68 & 280 & 115 & 2 & 0.65 & 6 & 97 & 278 & 118 & 1 & 0.824 \\
\hline $\mathrm{L} 1.4$ & 35 & 68 & 280 & 115 & 2 & 0.65 & 6 & 97 & 278 & 118 & 1 & 0.824 \\
\hline \multicolumn{13}{|c|}{ (Pair 2) Normal:Intrusion $=91: 409$} \\
\hline L1.1 & 3 & 88 & 263 & 144 & 2 & 0.828 & 0 & 91 & 216 & 193 & 0 & 0.932 \\
\hline $\mathrm{L} 1.2$ & 39 & 52 & 294 & 113 & 2 & 0.55 & 7 & 84 & 294 & 113 & 2 & 0.742 \\
\hline L1.3 & 39 & 52 & 292 & 115 & 2 & 0.554 & 7 & 84 & 294 & 113 & 2 & 0.742 \\
\hline L1.4 & 39 & 52 & 290 & 117 & 2 & 0.558 & 7 & 84 & 290 & 117 & 2 & 0.75 \\
\hline \multicolumn{13}{|c|}{ (Pair 3) Normal:Intrusion $=108: 392$} \\
\hline L1.1 & 9 & 99 & 234 & 154 & 4 & 0.926 & 5 & 103 & 193 & 198 & 1 & 1.02 \\
\hline L1.2 & 45 & 63 & 273 & 115 & 4 & 0.632 & 10 & 98 & 273 & 115 & 4 & 0.842 \\
\hline L1.3 & 45 & 63 & 273 & 115 & 4 & 0.632 & 10 & 98 & 273 & 115 & 4 & 0.842 \\
\hline L 1.4 & 45 & 63 & 273 & 115 & 4 & 0.632 & 10 & 98 & 273 & 115 & 4 & 0.842 \\
\hline
\end{tabular}

Table 4. L1 model generalization $\left(\mathrm{L} 1.1 \sim 4, G_{i n}=0\right)$.

The utility of the unknown subspace splitting step. L1.1 generalization without the range merging step is $\mathrm{L} 0$, which has no generalization at all. Comparing the detection results in Table 4 and Table 2, it is apparent that the generalization led to by the unknown subspace splitting step is useful to identify more instances, and significantly so for intrusive behaviors.

The difference between $\mathbf{L 1 . 2 / 3 / 4}$. The new false negatives caused by L1 generalization is negligible in all three sample pairs (with 1,2 and 3 additional ones). Overall, L1.2/3/4 have little difference on the detection results.

In summary, L1 generalization with $\mathrm{L} 1.2 / 3 / 4$ and range merging is useful but the detection results are not sensitive to the splitting strategies. Therefore, we arbitrarily select L1.4 with $G_{i n}=0$ in the following experiments. 


\subsection{Evaluating L2 Model Generalization}

Figures 2 and 3 list the evaluation results highlighting the influence of grouping and mutation opcrations on intrusion detection. In both figures, wc only illustrate $\#_{N A}, \#_{I A}$ and $\#_{I N}$ but the numbers of $\#_{N N}$ and $\#_{I I}$ can be deduced with ease since the total of normal and intrusive behaviors remains constant.

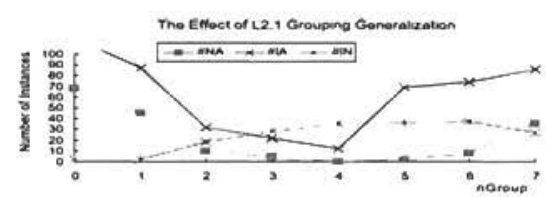

(a) Pair 1 (Normal:Intruxion=103:397).

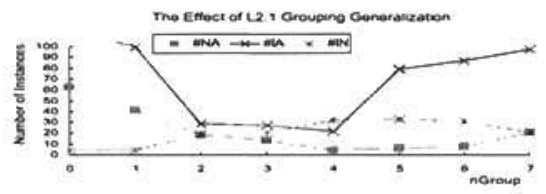

(c) Pair 3 (Normal:Intrusion=108:392).

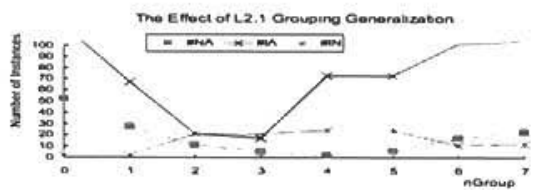

(b) Pair 2 (Normal:Intrusion $=91: 409$ ).

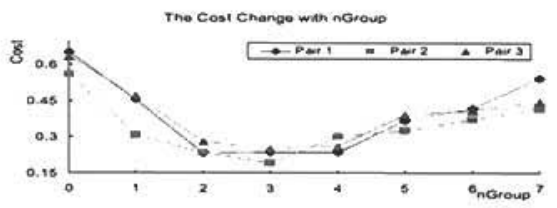

(d) Overall Performance w.r.t. nGroup

Fig. 2. L2 generalization-grouping (nMutate $=0$ ).

L2.1 grouping generalization. As indicated in Figure 2, the grouping operation enhances intrusion detection, and the detection performance on the three samples have the same characteristics. Specifically, the overall detection performance improves because of a reduction in the detection cost. With the increase of $n$ Group, $\#_{N N}$ and $\#_{I I}$ increase while $\#_{N A}$ and $\#_{I A}$ decrease, all of which are desirable. One negative aspect of grouping generalization is the increase of $\#_{I N}$ with the increase of $n$ Group.

Overall, the generalization from the grouping mechanism is uscful for intrusion detection even though it will lead to a few more false negatives. We choose $n$ Group $=3$ in the following experiments.

L2.2 mutation generalization. In Figure 3, the improvements caused by L2.2 mutation generalization is not that significant as L2.1 or L1 generalization. The decreased extent of false positives, $\#_{N A}$, is neutralized by the increased extent of false negatives, \#IN. This fact is also reflected by the overall detection cost in subfigure 3.d, which is reduced only by a very small extent. The mutation opcration will further worsen the negative aspects in grouping generalization.

In our casc study, the L2.2 mutation generalization is useful but it is not that significant. We select $n M$ utate $=5$ in evaluating L3 model generalization.

\subsection{Evaluating L3 Model Generalization}

In evaluating L3 generalization (Table 5), $n$ Group $=3$ and $n M u t a t e=5$. In the sample pair 1, all the normal behaviors are identificd correctly, and most intrusions are also identified correctly $(88.7 \%=352 / 397)$. In pair $1 / 2 / 3$, most normal bchaviors can be identificd correctly with fewer false positives (i.c., \# $\#_{A}$, which decreases with more gencralization) after the model generalization (from L1 to 


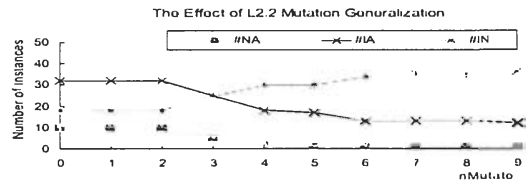

(a) Pair 1 (Normal:Intrusion=103:397)

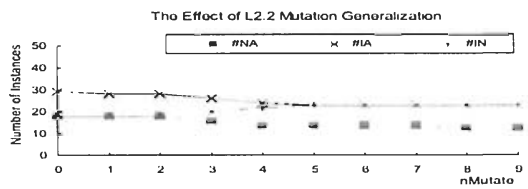

(c) Pair 3 (Normal:Intrusion=108:392).

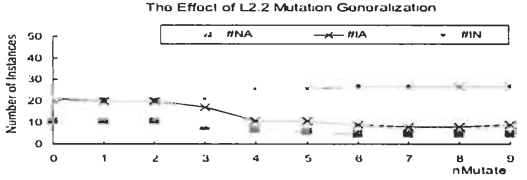

(b) Pair 2 (Normal:Intrusion=91:109).

The Cost Change with nMutate

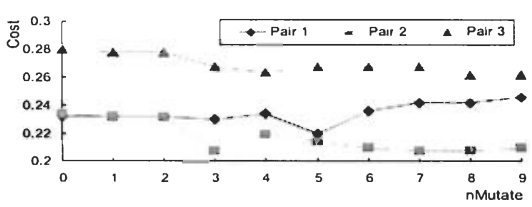

(d) Overall Performance w.r.t nMutate

Fig. 3. L2 generalization-mutation (nGroup=3).

\begin{tabular}{|l||c|c|c|c|c|c|}
\hline Sample & $\#_{N N}$ & $\#_{N A}$ & $\#_{I I}$ & $\#_{I A}$ & $\#_{I N}$ & cost \\
\hline Pair 1 & 103 & 0 & 352 & 4 & 41 & 0.254 \\
Pair 2 & 89 & 2 & 375 & 9 & 25 & 0.18 \\
Pair 3 & 105 & 3 & 353 & 2 & 37 & 0.244 \\
\hline
\end{tabular}

Table 5. L3 generalization (nGroup $=3$, nMutate $=5$ ).

L3). In contrast, even though more intrusive behaviors are identified correctly as well with more generalization, the false negatives (i.e., $\#_{I N}$ ) increase to a large extent (in comparison with Table 2).

\subsection{The Implications of Model Generalization}

In summary, model generalization is necessary for intrusion detection for identifying more behaviors correctly. The significance of every level of model generalization for intrusion detection is summarized in Table 6.

\begin{tabular}{|l|cc|l|}
\hline Levels & FP FN & Utility \\
\hline L0, L1.1 & - & - & $\begin{array}{l}\text { they act as an evaluation baseline to indicate whether model generalization } \\
\text { is necessary for intrusion detection. We also found that most intrusions are } \\
\text { identified even without generalization. }\end{array}$ \\
\hline L1.2/3/4 & $\downarrow$ & - & $\begin{array}{l}\text { They improve the detection performance in our case study, significantly for } \\
\text { intrusive behaviors. Most importantly, they lead to only a few more false neg- } \\
\text { atives. Their difference are negligible. }\end{array}$ \\
\hline $\begin{array}{l}\text { Range } \\
\text { Merging } \\
\text { in L1 }\end{array}$ & $\downarrow$ & - & $\begin{array}{l}\text { It is very useful to infer the statuses for normal behaviors, but it contributes } \\
\text { less in identifying intrusive behaviors. Another good point is that it does not } \\
\text { lead to more false negatives. }\end{array}$ \\
\hline L2.1 & $\downarrow$ & $\uparrow$ & $\begin{array}{l}\text { The identification capability is significantly lifted with decreasing anomalies. } \\
\text { However, there is an optimal value for the number of grouping points, which } \\
\text { should be determined in advance. }\end{array}$ \\
\hline L2.2 /L3 & $\downarrow$ & $\uparrow$ & $\begin{array}{l}\text { The identification capability is slightly lifted with decreasing anomalies. But } \\
\text { the increase of false negatives is so large that we should neglect the increase } \\
\text { of identification capabilities. }\end{array}$ \\
\hline
\end{tabular}

Table 6. The significance of different levels of model generalization. The symbol ' $\downarrow$ ' represents 'decrease' and the symbol ' $\uparrow$ ' represents 'increase'. '-' denotes that it will not affect the parameter. 


\section{Conclusions and Future Work}

In this paper, we designed a formal framework to evaluate the effect of various model generalization on intrusion detection in accordance with the reasonableness of its underlying assumptions. In a case study, we applied it to identify the implications of model generalization. We found that L1 generalization is generally useful to identify more 'novel' behaviors, especially for normal behaviors. L2.1 generalization will benefit intrusion detection by significantly improving the identification capability with slight increase of false negatives. The gains and losses from applying L2.2 and L3 generalization should be considered seriously under different application scenarios.

Even though our evaluation framework is generally applied to most scenarios for intrusion detection, it should be pointed out that our conclusions are only based on our case study on a typical dataset for intrusion detection. Our further work is to collect datasets to further evaluate the utility of model generalization in other areas, such as bioinformatics.

\section{References}

1. K.P. Anchor, J.B. Zydallis, G.H. Gunsch, and G.B. Lamont. Extending the computer defense immune system: Network intrusion detection with a multiobjective evolutionary programming approach. In ICARIS 2002: 1st International Conference on Artificial Immune Systems Conference Proceedings, 2002.

2. S.N. Chari and P. Cheng. BlueBox: A Policy-Driven, Host-based Intrusion Detection System. ACM Transaction on Infomation and System Security, 6(2):173-200, May 2003.

3. The KDD CUP 1999 Contest Dataset. As of january, 2006. http://wwwcse.ucsd.edu/users/elkan/clresults.html, 1999.

4. David E. Goldberg. Genetic algorithms in search, optimization, and machine learning. Addison-Wesley Pub. Co., 1989.

5. W. Lee and S.J. Stolfo. A framework for contructing features and models for intrusion detection systems. ACM Transactions on Information and System Security, 3(4):227-261, Nov. 2000.

6. Zhuowei Li, Amitabha Das, and Jianying Zhou. Theoretical basis for intrusion detection. In Proceedings of 6th IEEE Information Assurance Workshop (IAW), West Point, NY, USA, June 2005. IEEE SMC Society.

7. Shai Rubin, Somesh Jha, and Barton P. Miller. Automatic generation and analysis of nids attacks. In Proceedings of the 20th Annual Computer Security Applications Conference (ACSAC'04), pages 28-38, 2004.

8. Shai Rubin, Somesh Jha, and Barton P. Miller. Language-based generation and evaluation of nids signatures. In Proceedings of SES'05, pages 3-17, 2005.

9. Alfonso Valdes and Keith Skinner. Adaptive, model-based monitoring for cyber attack detection. In Proceedings of RAID'00, pages 80-92, October 2000.

10. Giovanni Vigna, William Robertson, and Davide Balzarotti. Testing networkbased intrusion detection signatures using mutant exploits. In Proceedings of CCS'04, pages 21-30, 2004.

11. David Wagner and Paolo Soto. Mimicry attacks on host-based intrusion detection systems. In Proceedings of CCS'O2, pages 255-264, 2002.

12. K. Wang and S.J. Stolfo. Anomalyous payload-based network intrusion detection. In Proceedings of RAID, 2004. 